\title{
Functional Foods for Cancer Therapeutics
}

\section{Pankaj Gupta*}

School of Medical and Allied Sciences, KR Mangalam University, Sohna Road, Gurgaon, Haryana, India

Functional foods have gained tremendous importance in recent times for their foreseen visualized role in the treatment of various pathologies such as cancer. Functional foods as the name indicates consist of foods that are of nutritional importance and are also involved in resolving the diseased state of the body. They have been reported for their beneficial role in cancer prevention and treatment. Several functional foods that have been obtained from various sources such as plants, animals and microbial sources are reported to possess noticeable anticancer effects with varying mechanisms of action viz. antimetastatic, induction of apoptosis, antiproliferative, antiangiogenic, scavenging of free radicals, inhibition of matrix metalloproteinases etc. Examples of functional foods that evinced anticancer potential include cereals, vegetables, beverages, dairy products, fish oil, beef, mushroom, probiotics etc.

The whole grain cereals that are consumed worldwide include wheat (Triticum aestivum L.), rice (Oryza sativa L.), maize (Zea mays ssp. mays L.), oats (Avena sativa L.), rye (Secale cereale L.) and barley (Hordeum vulgare L.). Whole grain cereals contain diverse components with anticancer potential which are located mostly in the germ and bran layers that are removed from the endosperm during refining. Bran is the hard outer layer of cereal grains that is profoundly rich in a myriad of healthy phytochemicals such as phenolics, flavonoids, glucans and pigments and has been observed to evince noticeable anticancer activity. Bitter melon (Momordica charantia L.) is a widely consumed vegetable throughout the world that comprises of several bioactive components such as polyphenols, flavonoids and saponins and has been reported to possess anticancer potential.

Thus, there are several examples of functional foods showing anticancer activity that may be exhibited via varying modes of action e.g., bitter melon inhibits proliferation of cancer cells, fish oil exhibits anticancer activity via inhibition of metastasis, several species of mushrooms especially Tricholoma matsutake act by inducing apoptosis and has shown beneficial effects in the treatment of oral cancer. Some functional foods have shown anticancer efficacy via inhibition of pathological angiogenesis such as green tea, red grapes etc. Several functional foods evinced anticancer activity via scavenging free radicals e.g., garlic, broccoli, green tea, soybean, tomato, carrot, cabbage, onion, cauliflower, red beets, cranberries, cocoa, blackberry, blueberry, red grapes, prunes and citrus fruits. Inhibition of matrix metalloproteinases by functional foods is yet another mechanism evolved for cancer treatment e.g., amla (Emblica officinalis) inhibit MMP-1, turmeric (Curcuma longa L.) inhibit MMP-2 and MMP14, Camellia sinensis inhibit MMP-1, MMP-2, MMP-3, MMP-7 and MMP-9 thereby exerting their anticancer effect. Thus, functional foods offer tremendous potential for the prevention and treatment of cancer and therefore may act as an alternative for cancer therapy.
${ }^{*}$ Corresponding author: Dr. Pankaj Gupta, Assistant Professor, School of Medical and Allied Sciences, KR Mangalam University, Sohna Road, Gurgaon-122 103, Haryana, India, Tel: +91-11-9818256122; E-mail: gupta.aiims@gmail.com

Received March 28, 2016; Accepted March 29, 2016; Published March 31, 2016

Citation: Gupta P (2016) Functional Foods for Cancer Therapeutics. Nat Prod Chem Res 4: e115. doi:10.4172/2329-6836.1000e115

Copyright: (c) 2016 Gupta P. This is an open-access article distributed under the terms of the Creative Commons Attribution License, which permits unrestricted use, distribution, and reproduction in any medium, provided the original author and source are credited. 